\title{
Cost-effectiveness of colorectal cancer screening in Germany: current endoscopic and fecal testing strategies versus plasma methylated Septin 9 DNA $^{1}$
}

Authors

Institutions
Uri Ladabaum ${ }^{1}$, Lourdes Alvarez-Osorio ${ }^{2}$, Thomas Rösch ${ }^{3}$, Bernd Brueggenjuergen ${ }^{2,4}$

Institutions are listed at the end of article. submitted

28. February 2014

accepted

17. March 2014

\section{Bibliography}

DOI http://dx.doi.org/

10.1055/s-0034-1377182

Published online: 6.6.2014

Endoscopy International Open

2014; 02: E96-E104

(C) Georg Thieme Verlag KG

Stuttgart · New York

E-ISSN 2196-9736

Corresponding author Uri Ladabaum, MD, MS Division of Gastroenterology and Hepatology,

Department of Medicine

Stanford University School of Medicine

300 Pasteur Drive,

Always Bldg Room M211

Stanford, CA, USA 94305-5187

Fax: +1-650-723-5488

uri.ladabaum@stanford.edu
Background and study aims: Colorectal cancer (CRC) screening strategies in Germany include guaiac-based fecal occult blood testing (gFOBT) starting at age 50 and a switch to colonoscopy at age 55 or continued gFOBT testing, but screening utilization is limited. Blood-based biomarkers, such as methylated Septin 9 DNA (' ${ }^{m}$ SEPT9), may improve screening rates. We performed a cost-effectiveness analysis of current and emerging CRC screening strategies in Germany.

Methods: Using a validated Markov model, we compared annual gFOBT for ages 50 through 54 followed by biennial testing until age 75 (FOBT) or by colonoscopy at ages 55 and 65 (FOBT/COLO $55,65)$, substitution of fecal immunochemical testing (FIT) for gFOBT (FIT, FIT/COLO 55,65), and annual or biennial plasma ${ }^{m}$ SEPT9 testing. We also considered persons who utilize only colonoscopy and varied age at colonoscopy utilization.

\section{Introduction}

\section{$\nabla$}

Colorectal cancer (CRC) is the second most common cancer in industrialized countries [1]. In Germany, it accounts for $12 \%$ of all cancer deaths in men, second only to lung cancer, and for $14 \%$ of all cancer deaths in women, second only to breast cancer [2]. Screening can decrease CRC-related mortality through cancer prevention by polypectomy and the removal of flat lesions and through early detection of CRC [3-9]. In Europe, CRC screening strategies and survival rates differ widely between countries [10]. Germany has in-

\footnotetext{
${ }^{1}$ Contributorship: conception and design, U. L.; analysis and interpretation of data, U. L., L. A., B. B.; drafting the article or revising it critically for important intellectual content, U. L., L. A., T. R., B. B.; final approval of the version to be published, U. L., L. A., T. R., B. B.

Funding sources: unrestricted research grant from $A b-$ bott Molecular, Des Plaines, IL, USA. The sponsor had no role in the conduct of this study. No future research funding by the sponsor is pending for any author.
}

Results: The current strategies were more effective and less costly than no screening. FIT was more effective and less costly than ${ }^{m}$ SEPT9 testing. FIT/COLO 55,65 cost €12200 per quality-adjusted life-years gained in comparison with FIT. ${ }^{m}$ SEPT9-based screening was cost-effective in comparison with no screening but was dominated by other cost-saving strategies. Differential screening utilization and adherence greatly affected incremental results between strategies. In probabilistic analyses, FIT was preferred in $49 \%$ and FIT/COLO 55,65 in 47\% of iterations.

Conclusion: Currently available CRC screening strategies in Germany, including hybrid fecal testing/colonoscopy, are likely to be cost-saving. Current strategies appear superior to ${ }^{m}$ SEPT9-based screening. The impact of blood-based biomarkers is likely to depend on utilization and adherence as much as on test performance characteristics and cost.

stituted an early detection program based on opportunistic screening, with limited attendance rates to date $[11,12]$.

A panel of experts has proposed CRC screening guidelines for Germany, taking into account current evidence [12]. Based on the determination of the Federal Standing Committee (GBA), which decides what is reimbursed by German statutory health insurance companies covering $85 \%$ of the German population, guaiac-based fecal occult blood testing (gFOBT) is covered starting at age 50 , and twice-in-a-lifetime screening colonoscopy is covered starting at age 55 [13]. Therefore, the current strategies that are recommended and fully covered by statutory health insurance in Germany include annual gFOBT for ages 50 through 54 , followed by biennial gFOBT or by colonoscopy starting at age 55 and repeated in 10 years. The German Guidelines for Colorectal Carcinoma consider colonoscopy the preferred test [12]. 
Despite these recommendations, the majority of Germans older than 50 years are not screened. In 2007-2008, 38\% of eligible women and $29 \%$ of eligible men older than 55 years of age underwent FOBT. The rate of colonoscopy utilization is lower. Between 2003 and 2008, approximately 16\% of eligible persons had undergone a screening colonoscopy [14], but more than $40 \%$ of persons older than 50 years of age had undergone a screening or diagnostic colonoscopy at least once by 2009 [15]. Lack of convenience and reservations about invasive methods are the leading reasons for this lack of adherence [14].

Blood-based biomarkers of colorectal neoplasia may have the potential to improve population screening rates. Promising markers are emerging [16], including plasma methylated Septin 9 DNA ( ${ }^{m}$ SEPT9) [17-19]. A large prospective trial in the United States and Germany recently demonstrated the test performance characteristics of a plasma ${ }^{m}$ SEPT9 assay for colorectal neoplasia in average-risk persons undergoing screening colonoscopy, with sensitivity for CRC of $48 \%$ (95\%CI $32-64 \%$ ) and specificity of $92 \%$ (95\%CI 90-93\%) when two replicates were used, and sensitivity for CRC of $64 \%$ (95\%CI $48-79 \%$ ) and specificity of $88 \%$ (95\%CI $86-90 \%$ ) in a post hoc three-replicate emulation [20]. A commercial assay for this marker is now available in Germany.

We used decision analytic modeling to perform a health economic evaluation of CRC screening in Germany, motivated by the recent establishment of Germany's unique CRC screening program and the emergence of novel biomarkers. We compared the strategies recommended in Germany, based on gFOBT and colonoscopy, with the substitution of gFOBT by a fecal immunochemical test (FIT) and with the use of ${ }^{m}$ SEPT9 as an illustration of emerging blood-based biomarkers.

\section{Methods}

$\nabla$

\section{Overview of decision analytic model}

We developed a model for Germany based on our previously published decision analytic model for the United States [21]. The model's previous validations [21,22] against the Minnesota Colon Cancer Control Study [3, 4] and three randomized controlled trials of sigmoidoscopy [7-9] and a model schematic (Appendix Fig.2) are presented in the Appendix. For this analysis, the natural history CRC incidence and mortality by age in the absence of screening were calibrated to year 2000 data from the Munich Cancer Registry (before the German national screening program was instituted) [23], and all-cause mortality by age was derived from the German national mortality table 2007-2009 [24] (Appendix Fig. 1). Because it can be debated which year's data are optimal for calibration, we tested the model with the original United States-based calibration and found that compared with the year 2000 German-based calibration, the cost-effectiveness analyses results did not differ with respect to ranking of strategies or dominance between strategies, and that while incremental cost-effectiveness ratios changed some, the differences were not substantial enough to affect interpretation or conclusions. Cost inputs were derived from a literature review [25-27], the doctor's fee scale and procedure reimbursement (Einheitlicher Bewertungsmaßstab: EBM) catalogue 2011 for office-based physicians with an EBM point value of $€ 0.035$ [28], German Diagnostic Related Group (DRG) codes for hospitalizations [29], and expert consultations (Appendix).

The health states in our model are as follows: normal; small polyp ( $<1 \mathrm{~cm}$ ); large polyp ( $\geq 1 \mathrm{~cm})$; CRC at localized, regional, or disseminated stages; and dead (Appendix Fig. 2). The model uses the staging system of the U.S. Surveillance, Epidemiology, and End Results (SEER) Program. SEER coding guidelines consider stage IIA as localized and stage IIB as regional disease. Data for Germany were converted to the SEER staging system.

\section{The PRESEPT trial and methylated Septin 9 DNA assays} PRESEPT (PRospective Evaluation of SEPTin) was a prospective multicenter study conducted in the United States and Germany that examined the performance for CRC detection of a polymerase chain reaction-based assay for ${ }^{m}$ SEPT9 in the plasma of average-risk persons undergoing screening colonoscopy [20]. Two plasma aliquots per person were initially tested (2-well assay), and a third aliquot of remaining DNA was tested post hoc to emulate a 3-well assay [20]. A 3-well assay is now available in Germany.

\section{Traditional and novel potential screening strategies,} and surveillance

We modeled the currently recommended and covered CRC screening strategies in Germany: gFOBT yearly for ages 50 through 54 and then every 2 years for ages 55 through 75 (FOBT), and hybrid strategies with FOBT and colonoscopy starting at age 55 (FOBT/COLO 55,65) or 60 (FOBT/COLO 60,70) [12, 13]. We compared these strategies with analogous strategies substituting FIT for gFOBT (FIT, FIT/COLO 55,65, and FIT/COLO 60,70). Different FITs are available, and test performance varies by cutoffs for positivity. We used a representative base case derived from published literature [30-32] and explored ranges in sensitivity analyses. To reflect persons who undergo only colonoscopy and not fecal testing, we modeled twice-in-a-lifetime colonoscopy at ages 55 and 65 (COLO 55,65) or ages 60 and 70 (COLO 60,70). Finally, we investigated strategies based on a 2-well or 3-well methylated Septin 9 DNA assay ( ${ }^{m}$ SEPT9-2well, ${ }^{m}$ SEPT9-3well) at varying test intervals. The ${ }^{m}$ SEPT9-3well strategies are meant to illustrate the potential improvement over the "mSEPT9-2well strategies, but we acknowledge that the ${ }^{m}$ SEPT9-3well model inputs reflect a post hoc analysis in the PRESEPT trial.

Screening was superimposed on the natural history module. Screening and surveillance were offered to persons at average risk for CRC from the ages of 50 to 75 years. If any screening test result was positive, then colonoscopy was offered. If the colonoscopy result was normal after a positive screening test result, the screening test was assumed to be a false-positive, and screening was resumed in 10 years with the primary screening strategy. With colonoscopy, polyps were removed and CRCs were biopsied if detected. In all strategies, surveillance colonoscopy was performed 3 years after the removal of a large adenoma $(\geq 1 \mathrm{~cm}), 5$ years after the removal of a small adenoma, and within 1 year of CRC diagnosis, then 3 years followed by every 5 years after CRC diagnosis [12,33].

\section{Septin 9-based screening: testing performance} and interval

We used the reported population-adjusted sensitivities of ${ }^{m}$ SEPT9-2well and ${ }^{m}$ SEPT9-3well from the PRESEPT trial [20] to estimate the assay's sensitivities for localized, regional, and disseminated CRC (Appendix Table 1).

In the PRESEPT trial, only one-time testing was performed. Before an ${ }^{m}$ SEPT9-2well or ${ }^{m}$ SEPT9-3well screening program could be compared with the alternative strategies, a testing interval had to be selected. We assumed that the sensitivities of fecal and 
blood-based tests were not affected by previous negative results. We examined screening with ${ }^{m}$ SEPT9-2well and ${ }^{m}$ SEPT9-3well at progressively shorter intervals, as we have described previously [34], and selected screening intervals that appeared to be reasonably cost-effective by traditional standards $(<25,000$ euros per quality-adjusted life-year [QALY] gained). We present results for yearly blood-based testing ( ${ }^{m}$ SEPT9-3well q1, mSEPT9-2well q1) as well as for blood-based testing every 2 years ( ${ }^{m}$ SEPT9-3well

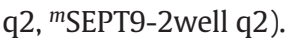

\section{Cost inputs}

The derivation of cost inputs for Germany in year 2011 euros is described in the Appendix. The cost of the ${ }^{m}$ SEPT9 assay in Germany is currently approximately $€ 150$.

\section{Clinical and economic outcomes}

The principal model outputs were QALYs and costs per person $[35,36]$. Future QALYs and costs were discounted by $3 \%$ annually [37]. The seemingly small differences in QALYs between strategies result from the fact that gains of several years among a small fraction of people are averaged over the entire screened population and future years are discounted; this should not be interpreted as indicating that the clinical outcome differences between strategies are negligible [21,22]. Reported health state utilities for CRC by stage were used to calculate QALYs by applying these for 5 years after CRC diagnosis. For each strategy, we estimated CRC cases by stage, CRC-related deaths, and colonoscopy demand in a cohort of 100000 persons.

\section{Cost-effectiveness analyses}

Analyses from the perspective of a health care payer were performed in TreeAge Pro (TreeAge Software, Inc., Williamstown, MA, USA) and in Excel 2003 (Microsoft Corporation, Redmond, WA, USA). Incremental cost-effectiveness ratios (ICERs) were calculated $[35,36]$.

We first performed analyses reflecting persons who undergo and adhere to screening (maximum efficacy). Second, because utilization and adherence are likely to differ between strategies, although data on these parameters are not available for all strategies, we explored in detail multiple scenarios reflecting imperfect and differential utilization and per-cycle screening adherence (potential effectiveness). Analyses focused on variable utilization compare populations with different proportions of "regularly adherent" versus "never-adherent" persons, and analyses focused on variable per-cycle adherence examine persons who are "irregularly adherent." Detailed results for all strategies are presented in figures and tables, but for ease of understanding, the text emphasizes the most salient comparisons.

One-way sensitivity analyses were performed on all model inputs. Threshold analyses were performed on influential variables. To estimate the uncertainty of our projections, a Monte Carlo simulation with 1000 trials was performed. We used beta distributions for probabilities derived from means, standard deviations, and ranges in the literature [38]. Costs of screening were varied as a set by the same factor (common factor) within a range of $20 \%$ of the base case value. Costs of care were varied as a set by a different common factor within the same range.

\section{Results}

$\nabla$

\section{Testing interval for strategies based on mSEPT9}

Because the incremental cost per QALY gained with yearly ${ }^{m}$ SEPT9-3well or ${ }^{m}$ SEPT9-2well compared with screening every 2 years was relatively cost-effective ( Table 1 ), and because all four of these strategies were cost-effective compared with no screening ( $\bullet$ Table 1 ), we present results for ${ }^{m}$ SEPT9-based testing yearly as well as every 2 years.

\section{Base case: Effectiveness with optimal utilization} and adherence

All screening strategies decreased CRC incidence, shifted the stages of CRC at diagnosis toward earlier stages, and decreased CRC-related mortality ( $\bullet$ Table 1 ). CRC incidence reductions resulted from adenoma detection and removal. Shifts in CRC stage resulted from detection of early CRC. Decreases in CRC mortality resulted from both prevention and early detection.

Assuming optimal screening utilization and adherence, the greatest reductions in CRC incidence and mortality compared with no screening were observed with the hybrid strategies of FIT and colonoscopy, then gFOBT and colonoscopy ( Table 1 ). The mSEPT9-based strategies resulted in less substantial CRC incidence reductions, reflecting their lower sensitivities for adenomas.

Beyond CRC incidence, CRC stage and patient age at diagnosis were also important determinants of life expectancy. When these were considered in addition to CRC incidence, the greatest gains in life expectancy were observed with FIT/COLO 55,65, followed by FIT alone ( $\bullet$ Fig. 1, $\bullet$ Table 1 ).

\section{Base case: cost-effectiveness and colonoscopy demand with optimal utilization and adherence}

All strategies based on fecal tests and/or colonoscopy and ${ }^{m}$ SEPT9-3well q2 cost less than no screening (i.e., they were cost-saving), making them dominant over no screening because they were also more effective $($ Fig. $1, \bullet$ Table 1 ). The remaining ${ }^{m}$ SEPT9-based strategies were not cost-saving, but they were cost-effective compared with no screening, at costs of $€ 600$ to $€ 3600$ per QALY gained ( $\bullet$ Fig. 1, $\bullet$ Table $\mathbf{1}$ ).

In incremental comparisons between all strategies, several strategies were dominated by other strategies that had greater effectiveness and lower costs ( $\bullet$ Fig. 1, $\bullet$ Table 1 ). The two strategies that emerged as preferred after the dominated strategies had been excluded were FIT, which was dominant over no screening and nearly all other strategies, and FIT/COLO 55,65, which displayed an ICER of $€ 12200$ per QALY gained compared with FIT ( Table 1). Colonoscopy demand was substantially lower with FIT than with hybrid strategies or annual ${ }^{m}$ SEPT9-based strategies ( Table 1 ).

\section{Imperfect screening utilization and adherence}

Differential screening utilization, which refers to the fraction of a population that participates in screening, affected the comparisons between strategies. For example, if comparable cohorts were offered FIT or ${ }^{m}$ SEPT9-3well q2, and the relative utilization rate of FIT was 70\% versus that of ${ }^{m}$ SEPT9-3well q2, then FIT shifted to being comparatively less effective instead of more effective than ${ }^{m}$ SEPT9-3well q2, and ${ }^{m}$ SEPT9-3well q2 had an incremental cost of $€ 59600$ per QALY gained compared with FIT. The ICER of ${ }^{m}$ SEPT9-3well q2 compared with FIT decreased to $€ 26300$ per 


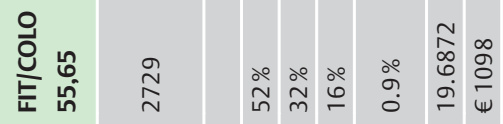

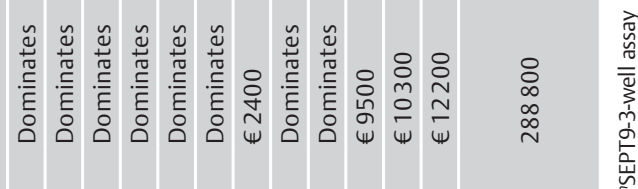

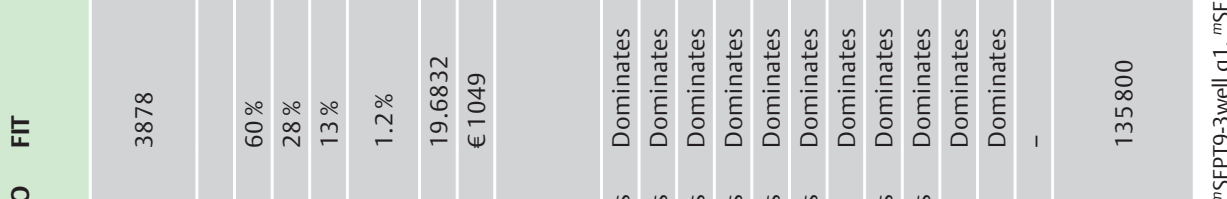

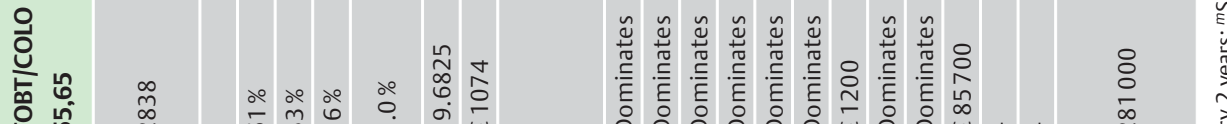

空

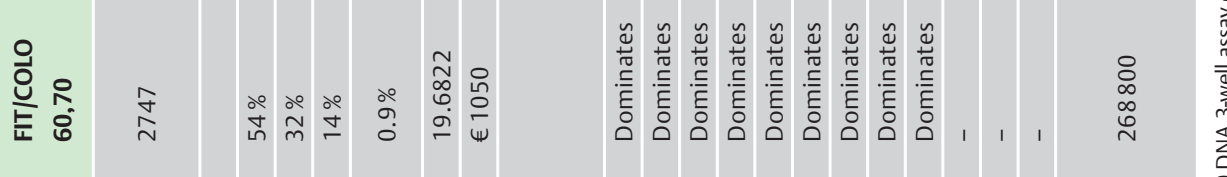

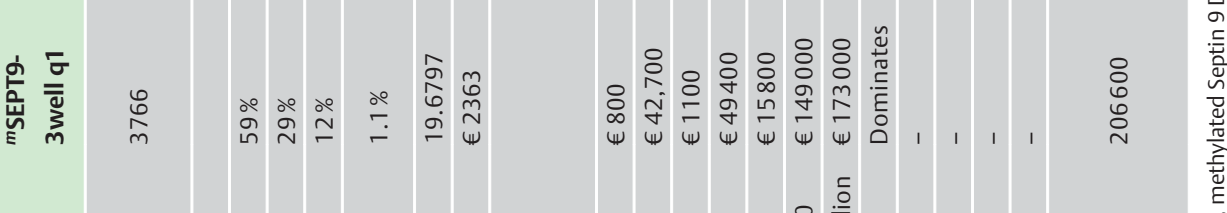

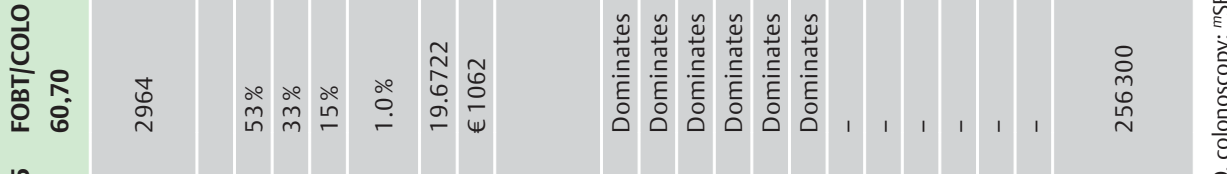

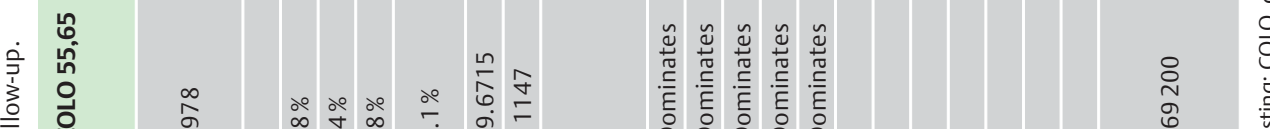

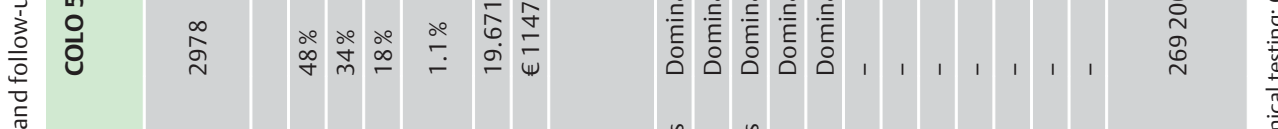

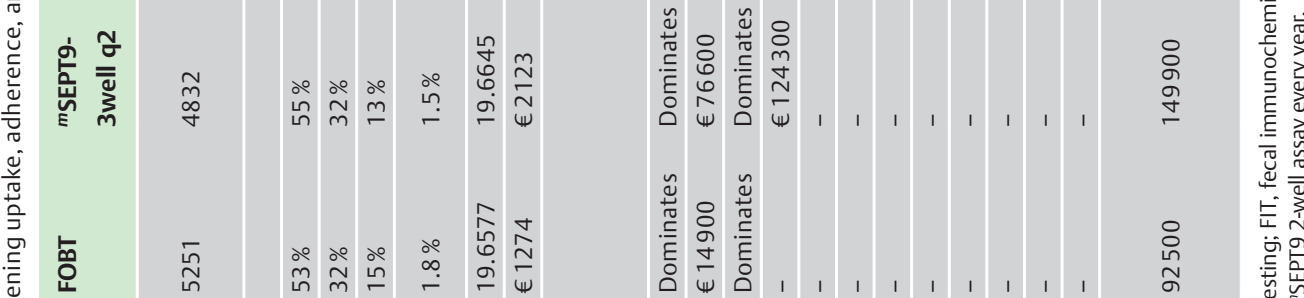

品

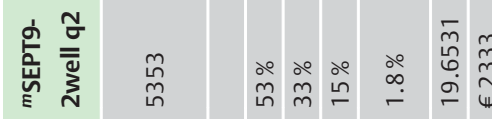

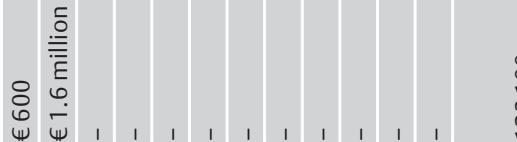

这家

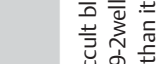

웅

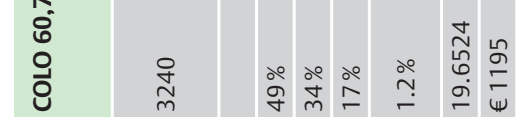

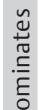

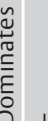

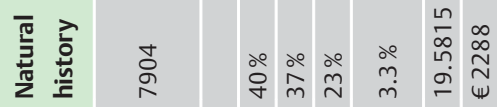

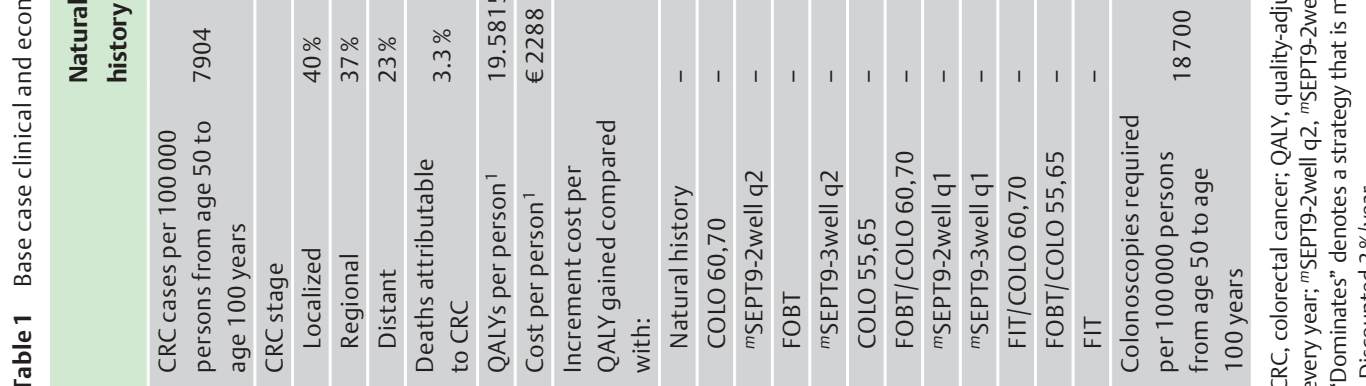

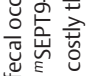

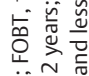

\&

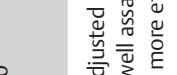

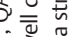

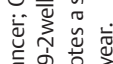




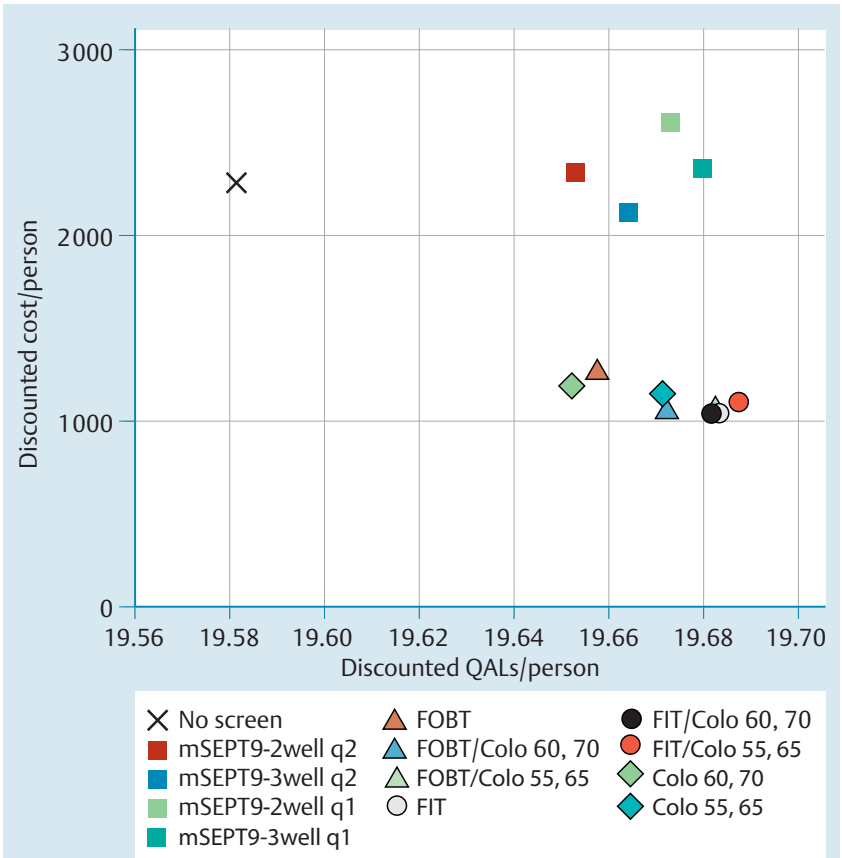

Fig. 1 Discounted mean quality-adjusted life-years (QALYs) per person and costs per person for the screening strategies in the base case. The strategies using gFOBT (guaiac-based fecal occult blood testing), FIT (fecal immunochemical testing), and/or COLO (colonoscopy) were all more effective and less costly than no screening. Among the mSEPT9 (methylated Septin 9 DNA)-based strategies, mSEPT9-3well q2 (3-well assay every 2 years) was more effective and less costly than no screening, while ${ }^{m}$ SEPT93 well q1 (3-well assay every year), mSEPT9-2well q2 (2-well assay every 2 years), and mSEPT9-2well q1 (2-well assay every year) were all highly costeffective but not cost-saving.

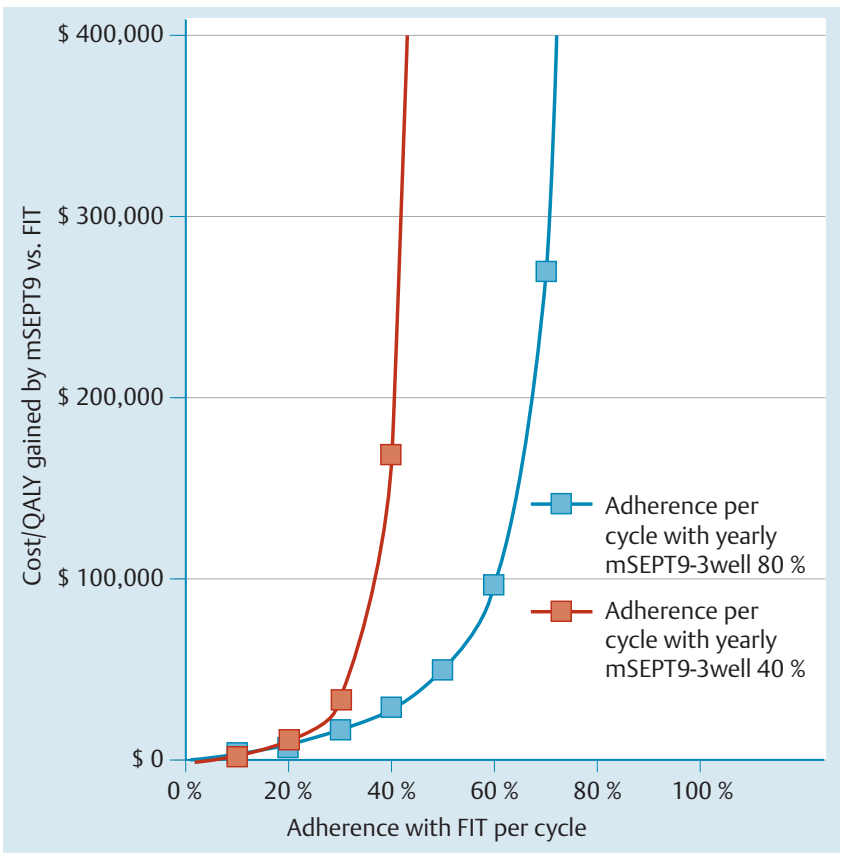

Fig. 2 Sensitivity analysis on per-cycle adherence rates: ${ }^{\text {SSEPT9-3well q1 }}$ (methylated Septin 9 DNA 3-well assay every year) versus FIT (fecal immunochemical testing yearly for ages $50-54$ years, then every 2 years for ages $55-75$ years). Assuming $80 \%$ per-cycle adherence with ${ }^{m}$ SEPT9-3well q1, this strategy cost $€ 28800$ per quality-adjusted life-year gained compared with FIT with $40 \%$ per-cycle adherence.

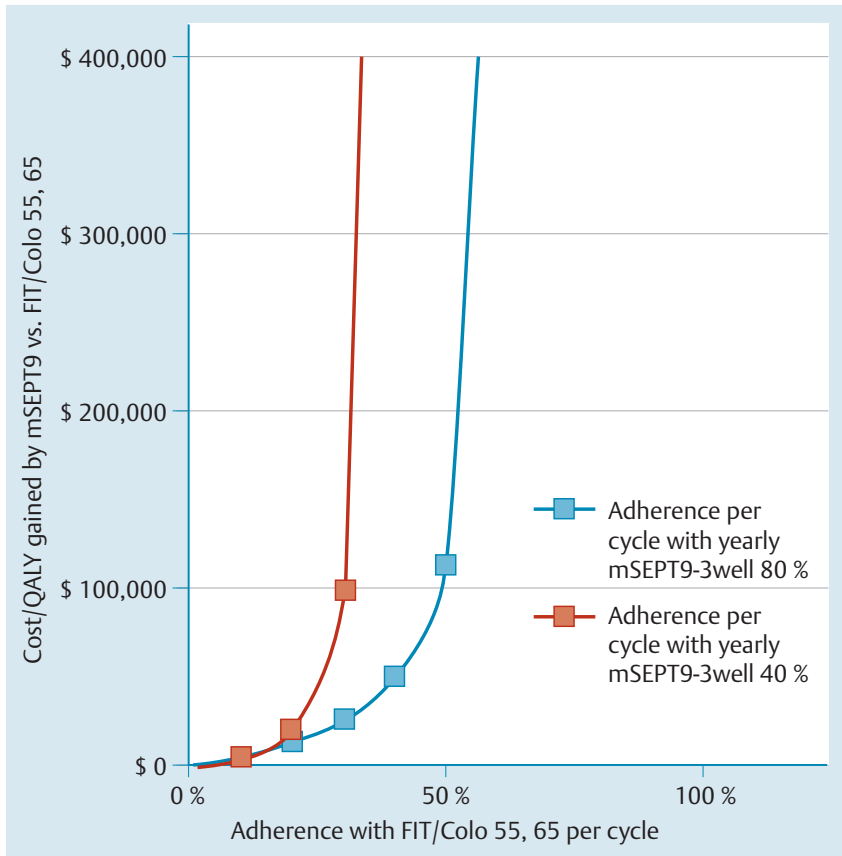

Fig. 3 Sensitivity analysis on per-cycle adherence rates: mSEPT9-3well q1 (methylated Septin 9 DNA 3-well assay every year) versus FIT/COLO 55,65 (fecal immunochemical testing yearly for ages $50-54$ years and colonoscopy at ages 55 and 65). Assuming $80 \%$ per-cycle adherence with ${ }^{m}$ SEPT93 well q1, this strategy cost $€ 50000$ per quality-adjusted life-year gained compared with FIT/COLO 55,65 with $40 \%$ per-cycle adherence.

QALY when the relative FIT uptake rate was $60 \%$ versus that of mSEPT9-3well q2.

Differential rates of per-cycle adherence among persons utilizing screening, which refers to the probability of accepting a screening offer in a particular cycle of a screening program, also affected the comparisons between strategies. For example, assuming $80 \%$ per-cycle adherence with ${ }^{m}$ SEPT9-3well q1, this strategy had ICERs of $€ 28800$ per QALY gained compared with FIT with $40 \%$ per-cycle adherence, and $€ 50000$ per QALY gained compared with FIT/COLO 55,65 with $40 \%$ per-cycle adherence (๑ Fig.2, $\odot$ Fig.3).

\section{Sensitivity analyses}

Assuming optimal uptake and adherence, comparisons between strategies were affected by test performance characteristics and costs, but the costs of CRC care and complication rates had negligible impact, and they did not affect the rankings between strategies, including ${ }^{m}$ SEPT9-based screening compared with fecal testing ( $\bullet$ Table 2 ). FIT remained dominant over the ${ }^{m}$ SEPT9based strategies even at a test cost for ${ }^{m}$ SEPT9 of $€ 10$. Assuming a best-case scenario for ${ }^{m}$ SEPT9-3well q1 test performance characteristics based on the upper bounds of reported confidence intervals (sensitivities of $15 \%$ for small polyp, $19 \%$ for large polyp, $75 \%$ for localized cancer, and $100 \%$ for regional cancer; specificity 90\%), this strategy's effectiveness (mean 19.6799 QALY per person) approached that of the most effective strategies ( $\bullet$ Table 1 ), but a test cost of $€ 30$ was required to achieve a total cost (mean $€ 1165$ per person) that approximated the costs of the most effective strategies ( $\bullet$ Table 1 ). Even with these parameters, the FIT-based strategies and FOBT/COLO 55,65 remained more effective and less costly, and therefore dominant, compared with ${ }^{m}$ SEPT9-3well q1. 
Table 2 One-way sensitivity analyses focusing on ${ }^{\mathrm{m} S E P T 9-b a s e d ~ s c r e e n i n g ~ a n d ~ f e c a l ~ t e s t i n g . ~}$

\begin{tabular}{|c|c|c|c|c|c|c|c|c|}
\hline \multirow[t]{2}{*}{ Variable } & \multirow{2}{*}{$\begin{array}{l}\text { Base case } \\
\text { value }\end{array}$} & \multirow{2}{*}{$\begin{array}{l}\text { Value in sensi- } \\
\text { tivity analysis }\end{array}$} & \multicolumn{2}{|c|}{$\begin{array}{l}\text { mSEPT9-3well q1 vs. natural } \\
\text { history }\end{array}$} & \multicolumn{2}{|c|}{ mSEPT9-3well q1 vs. FOBT } & \multicolumn{2}{|c|}{ mSEPT9-3well q1 vs. FIT } \\
\hline & & & $\begin{array}{l}\text { Incremental } \\
\text { QALYs }^{1}\end{array}$ & $\begin{array}{l}\text { Incremental } \\
\text { cost per QALY } \\
\text { gained }^{1}\end{array}$ & $\begin{array}{l}\text { Incremental } \\
\text { QALYs' }^{1}\end{array}$ & $\begin{array}{l}\text { Incremental } \\
\text { cost per QALY } \\
\text { gained }^{1}\end{array}$ & $\begin{array}{l}\text { Incremental } \\
\text { QALYs }^{1}\end{array}$ & $\begin{array}{l}\text { Incremental } \\
\text { cost per QALY } \\
\text { gained }^{1}\end{array}$ \\
\hline \multirow{2}{*}{$\begin{array}{l}\text { mSEPT9-3well } \\
\text { sensitivity for } \\
\text { small polyp/ } \\
\text { large polyp/ } \\
\text { localized CRC/ } \\
\text { regional CRC }\end{array}$} & $\begin{array}{l}0.10 / 0.14 / \\
0.51 / 0.75\end{array}$ & $\begin{array}{l}0.06 / 0.10 / \\
0.26 / 0.42\end{array}$ & 0.0876 & $€ 2260$ & 0.0115 & $€ 105500$ & -0.0181 & Dominated \\
\hline & & $\begin{array}{l}0.15 / 0.19 / \\
0.75 / 1.0\end{array}$ & 0.1056 & Dominates & 0.0295 & $€ 31200$ & 0.0040 & $€ 289200$ \\
\hline \multirow[t]{2}{*}{$\begin{array}{l}\text { mSEPT9-3well } \\
\text { specificity }\end{array}$} & 0.88 & 0.85 & 0.0975 & $€ 30$ & 0.0214 & $€ 47600$ & -0.0042 & Dominated \\
\hline & & 0.90 & 0.0987 & $€ 1300$ & 0.0226 & $€ 50700$ & -0.0030 & Dominated \\
\hline \multirow[t]{2}{*}{$\begin{array}{l}\text { mSEPT9-3well } \\
\text { cost }\end{array}$} & $€ 150$ & $€ 50$ & 0.0982 & Dominates & 0.0221 & $€ 9000$ & -0.0035 & Dominated \\
\hline & & $€ 100$ & 0.0982 & Dominates & 0.0221 & $€ 29200$ & -0.0035 & Dominated \\
\hline $\begin{array}{l}\text { FOBT sensitivity } \\
\text { for small polyp/ } \\
\text { large polyp/CRC }\end{array}$ & $\begin{array}{l}0.05 / 0.11 / \\
0.40\end{array}$ & $\begin{array}{l}0.05 / 0.09 / \\
0.25\end{array}$ & 0.0982 & $€ 800$ & 0.0360 & $€ 25300$ & -0.0035 & Dominated \\
\hline $\begin{array}{l}\text { FIT sensitivity } \\
\text { for small polyp/ } \\
\text { large polyp/CRC }\end{array}$ & $\begin{array}{l}0.10 / 0.24 / \\
0.70\end{array}$ & $\begin{array}{l}0.075 / 0.16 / \\
0.50\end{array}$ & 0.0982 & $€ 800$ & 0.0221 & $€ 49400$ & 0.0100 & $€ 112000$ \\
\hline $\begin{array}{l}\text { Colonoscopy } \\
\text { sensitivity for } \\
\text { small polyp/ } \\
\text { large polyp/CRC }\end{array}$ & $\begin{array}{l}0.85 / 0.90 / \\
0.95\end{array}$ & $\begin{array}{l}0.75 / 0.85 / \\
0.92\end{array}$ & 0.0962 & $€ 1200$ & 0.0219 & $€ 50100$ & -0.0034 & Dominated \\
\hline $\begin{array}{l}\text { Colonoscopy } \\
\text { bleeding/per- } \\
\text { foration rates }\end{array}$ & $\begin{array}{l}0.0016 / \\
0.00085\end{array}$ & $\begin{array}{l}0.008 / \\
0.00425 \\
\text { (5-fold } \\
\text { increase) }\end{array}$ & 0.0928 & $€ 1300$ & 0.0187 & $€ 60000$ & 0.0056 & Dominated \\
\hline \multirow[t]{2}{*}{ CRC care costs } & $\begin{array}{l}\text { See Appen- } \\
\text { dix Table } 1\end{array}$ & $50 \%$ decrease & 0.0982 & $€ 8700$ & 0.0221 & $€ 58500$ & -0.0035 & Dominated \\
\hline & & $50 \%$ increase & 0.0982 & Dominates & 0.0221 & $€ 40200$ & -0.0035 & Dominated \\
\hline
\end{tabular}

CRC, colorectal cancer; QALY, quality-adjusted life-year; FOBT, fecal occult blood testing; FIT, fecal immunochemical testing; mSEPT9-3well q1, methylated Septin 9 DNA 3-well assay every year.

"Dominates" denotes a strategy that is more effective and less costly than its comparator.

"Dominated" denotes a strategy that is less effective and more costly than its comparator.

${ }^{1}$ Discounted 3\%/year.

\section{Monte Carlo simulation}

Assuming optimal utilization and adherence, the preferred strategies at a willingness-to-pay threshold of $€ 25000$ per QALY gained were FIT in $49 \%$ of iterations, FIT/COLO 55,65 in $47 \%$ of iterations, and FOBT/COLO 55,65 in $4 \%$ of iterations. At a willingness-to-pay threshold of $€ 50000$ per QALY gained, FIT was preferred in $37 \%$ of iterations, FIT/COLO 55,65 in $62 \%$ of iterations, and FOBT/COLO 55,65 in $1 \%$ of iterations.

The median and $95 \%$ confidence intervals for the QALYs gained per person with each strategy and the incremental costs per QALY gained between strategies are shown in - Table 3. FIT and FIT/COLO 55,65 were the two remaining strategies when dominated strategies were excluded. Compared with no screening, FIT gained a median 0.0810 (95\%CI $0.0661-0.1025)$ QALY per person, and it was less costly (dominant) within the entire $95 \%$ confidence interval. Compared with FIT, FIT/COLO 55,65 gained a median 0.0028 (95\%CI $0.002-0.011)$ QALY per person, and it cost $€ 10200$ (95\%Cl Dominates - 446 000) per QALY gained.

\section{Discussion}

Our health economic evaluation of CRC screening in Germany explores the potential effectiveness and cost-effectiveness of current strategies that rely on gFOBT and colonoscopy, as well as the potential substitution of FIT for gFOBT and emerging bloodbased biomarkers, exemplified by a plasma ${ }^{m}$ SEPT9 assay [20]. The results have implications for screening in Germany specifically, but the novel insights provided on hybrid screening strategies have implications for CRC screening more broadly.

In recent years, national screening programs have been debated or instituted in multiple countries, and these are usually based on fecal tests or sigmoidoscopy. Colonoscopy is frequently used in the United States where there is no uniform national screening program [39], and it is recommended as the preferred screening test in Germany [12]. The currently recommended and covered strategies in Germany include a unique hybrid strategy that combines FOBT in the initial years, followed by colonoscopy in later years.

Our results illustrate the potential benefit of hybrid fecal test/colonoscopy strategies. In the base case, FIT/COLO 55,65 was the 
Table 3 Results of Monte Carlo simulation.

\begin{tabular}{|c|c|c|c|}
\hline Strategy & $\begin{array}{l}\text { Increment QALYs gained per person } \\
\text { vs. natural history, } \\
\text { median ( } 95 \% \text { confidence interval) }{ }^{1}\end{array}$ & $\begin{array}{l}\text { Increment cost per QALY gained } \\
\text { vs. natural history, } \\
\text { median ( } 95 \% \text { confidence interval) }{ }^{1}\end{array}$ & $\begin{array}{l}\text { Increment cost per QALY gained } \\
\text { vs. preceding strategy, } \\
\text { median ( } 95 \% \text { confidence interval) }{ }^{1}\end{array}$ \\
\hline COLO 60,70 & $0.0567(0.0470-0.0723)$ & Dominates (Dominates - Dominates) & Dominates (Dominates - Dominates) ${ }^{2}$ \\
\hline mSEPT9-2well q2 & $0.0574(0.0459-0.0728)$ & $€ 4200(€ 1200-€ 7900)$ & $€ 159000$ (Dominates - $€ 4.0$ million) \\
\hline FOBT & $0.0604(0.0464-0.0775)$ & Dominates (Dominates - Dominates) & Dominates (Dominates $-€ 1.6$ million) \\
\hline mSEPT9-3well q2 & $0.0668(0.0553-0.0825)$ & $€ 900$ (Dominates - $€ 3500$ ) & $€ 92000$ (Dominates - $€ 1.5$ million) \\
\hline COLO 55,65 & $0.0717(0.0600-0.0899)$ & Dominates (Dominates - Dominates) & Dominates (Dominates - $€ 872000$ ) \\
\hline FOBT/COLO 60,70 & $0.0724(0.0601-0.0903)$ & Dominates (Dominates-Dominates) & Dominates (Dominates - $€ 601000$ ) \\
\hline mSEPT9-2well q1 & $0.0731(0.0604-0.0928)$ & $€ 8000(€ 5100-€ 11400)$ & $€ 281000$ (Dominates - $€ 7.2$ million) \\
\hline mSEPT9-3well q1 & $0.0786(0.0656-0.0975)$ & $€ 4500(€ 2200-€ 7200)$ & Dominates (Dominates - $€ 158000$ ) \\
\hline FIT/COLO 60,70 & $0.0801(0.0665-0.1007)$ & Dominates (Dominates - Dominates) & Dominates (Dominates $-€ 6.3$ million) \\
\hline FOBT/COLO 55,65 & $0.0804(0.0677-0.0996)$ & Dominates (Dominates - Dominates) & Dominates (Dominates - $€ 127000$ ) \\
\hline FIT & $0.0810(0.0661-0.1025)$ & Dominates (Dominates - Dominates) & Dominates (Dominates - $€ 99700$ ) \\
\hline FIT/COLO 55,65 & $0.0841(0.0706-0.1050)$ & Dominates (Dominates - Dominates) & 10200 (Dominates - € 446000) \\
\hline
\end{tabular}

QALY, quality-adjusted life-year; FOBT, fecal occult blood testing; FIT, fecal immunochemical testing; COLO, colonoscopy; ${ }^{m}$ SEPT9-3well q2, methylated Septin 9 DNA 3-well assay every 2 years; ${ }^{m}$ SEPT9-3well q1, ${ }^{m}$ SEPT9 3 -well assay every year; ${ }^{m}$ SEPT9-2well q2, ${ }^{m}$ SEPT9 2-well assay every 2 years; ${ }^{m}$ SEPT9-2well q1, ${ }^{m}$ SEPT9 2-well assay every year.

"Dominates" denotes a strategy that is more effective and less costly than its comparator.

1 Discounted 3\%/year.

${ }^{2}$ Compared with natural history.

most effective strategy, followed closely by FIT ( $\bullet$ Table 1 ), and these were also the preferred strategies in the Monte Carlo simulation. The optimal choice between these strategies depends on society's willingness to pay per QALY gained, and on available endoscopic resources. The hybrid strategies take advantage of frequent noninvasive testing at the younger screening ages, with a high cumulative sensitivity over the 5 initial years for prevalent and incident CRCs and adenomas. Thereafter, following those who have had a negative FIT for 5 years with subsequent FIT every 2 years or colonoscopy at ages 55 and 65 , both seem to be reasonable options ( Table 1, Table 3 ). However, the demand for colonoscopy was substantially higher with hybrid strategies than with FIT ( $\bullet$ Table 1). Furthermore, differential utilization and adherence rates between strategies affected not only the absolute benefits and costs of a given strategy compared with no screening but also the incremental comparisons between strategies.

Some patients may utilize twice-in-a-lifetime colonoscopy beginning at age 55 without undergoing gFOBT starting at age 50 . Our analysis suggests that performing twice-in-a-lifetime screening colonoscopies at younger ages (e.g., ages 55 and 65) may be more effective and cost-effective than performing them at older ages (e.g., ages 60 and 70). However, FIT and all FIT-based or gFOBT-based hybrid strategies starting at age 50 were more effective than twice-in-a-lifetime colonoscopy alone starting at age 55 or later, assuming optimal adherence. These results highlight the differences between a program of frequent use of a test that is reasonably sensitive for CRC but less sensitive for adenomas on one-time testing (FIT) versus a program of twice-in-alifetime use of a test with higher sensitivities but a much longer testing interval in which neoplasia can develop or progress (COLO 55,65). The relative utilization for each program and the relative adherence with each test cycle, however, are both important determinants of a given program's ultimate effectiveness and cost-effectiveness [40], as discussed further below. It remains to be determined what a hybrid program's utilization and adherence over time will be.

At present, FIT is available in Germany, but its cost generally must be covered by patients themselves. Our results suggest that insurance coverage for FIT instead of gFOBT could improve clinical out- comes and prove cost-saving for persons who undergo fecalbased screening (FIT vs. FOBT, $\bullet$ Table 1) or fecal-based screening and then colonoscopy at later ages (FIT/COLO 60,70 vs. FOBT/ COLO 60,70; Table 1), and cost-effective for persons who undergo fecal-based screening and then colonoscopy at younger ages (FIT/COLO 55,65 vs. FOBT/COLO 55,65; • Table 1).

How might novel blood-based biomarkers fit into the landscape of CRC screening in Germany? A plasma ${ }^{m}$ SEPT9 assay is currently available in Germany, but patients must cover its cost. Multiple other biomarkers are under study [16]. Blood-based assays offer the theoretical advantage of minimal invasiveness and possibly greater acceptance by patients. With current test performance characteristics and cost, ${ }^{m}$ SEPT9-based screening may be less effective and cost-effective than the alternatives, assuming comparable utilization and adherence between all strategies. However, if screening with a blood test resulted in greater numbers of people adhering to screening, it could be more effective at the population level than the alternatives ( Fig.2, Fig.3). A blood-based assay with test performance characteristics matching the upper range of the confidence intervals that we assumed for the ${ }^{m}$ SEPT9-3well assay [20], if offered at a cost of approximately $€ 30$, could be competitive against FIT in Germany, even at high levels of utilization and adherence for both tests.

Our study has several strengths. Our decision analytic model has been validated $[21,22]$ against prospective, randomized clinical trials of FOBT $[3,4]$ and sigmoidoscopy [7-9]. The test performance characteristics for ${ }^{m}$ SEPT9-based screening were obtained from a prospective trial [20]. We have adapted our model to study CRC screening in Germany, which has unique features, including the recommended and covered strategies of gFOBT at yearly and then biennial intervals, and a hybrid screening strategy combining gFOBT and a later switch to colonoscopy. Our analyses provide novel insight into CRC screening in general, independently of the particular setting. Finally, our model provides a means to evaluate emerging blood-based biomarkers in the context of relatively low costs for established screening tests, including colonoscopy, as is the case in Germany.

We acknowledge some limitations. This is a modeling study, and the results depend directly on the model inputs and assumptions. We have assumed conditional independence between repeated 
rounds of testing, which may not be true [41], particularly regarding sensitivity for advanced adenoma (Appendix). This may overestimate cumulative program sensitivity, which may be particularly relevant for FIT- and ${ }^{m}$ SEPT9-based screening. Because the base case results for these strategies may overestimate their effectiveness, we have addressed program sensitivity by varying the sensitivities per lesion. While we explored uncertainties in all model parameters in sensitivity analyses, it must be appreciated that little is known about certain key parameters. For instance, no longitudinal data are available on the utilization of and adherence to current screening strategies (including hybrid strategies) over time in Germany. There are no data on these parameters for emerging blood-based biomarkers. Therefore, the scenarios we present for differential uptake and adherence must be taken as illustrations. The results of any modeling study must be interpreted with caution because modeling studies are not clinical trials. The advisability of starting colonoscopy screening at age 55 and not earlier, and the specific designs of the hybrid strategies, might be debated by some, but we chose to model these strategies as currently covered in Germany. Further data are needed on patient preferences, which affect utilization and adherence rates, and therefore the estimated effectiveness and cost-effectiveness, of hybrid and novel strategies. Our focus on specific comparisons and threshold analyses addresses the a priori aims of this study, but it does not imply that these strategies are preferred, especially in light of the uncertainties surrounding utilization and adherence. Finally, our CRC treatment cost inputs were not based on a cost-of-illness study, but instead on expert opinions and other sources.

In conclusion, the currently recommended and covered CRC screening strategies in Germany, including hybrid strategies of fecal-based testing and colonoscopy, are likely to be cost-saving. The place of emerging blood-based biomarkers such as ${ }^{m}$ SEPT9 among screening options in Germany will depend not only on their test performance characteristics and cost, but also on their utilization and longitudinal adherence over time compared with the accepted alternatives.

Competing interests: None current. U.L. consultant, Epigenomics (Aug 2007-Nov 2009)

\section{Institutions}

${ }^{1}$ Division of Gastroenterology and Hepatology, Department of Medicine, Stanford University School of Medicine, Stanford, CA, USA

${ }^{2}$ Boston Healthcare Associates International GmbH, Berlin, Germany

${ }^{3}$ Charité Medical University of Berlin, Virchow Clinic Campus, Berlin, Germany

${ }^{4}$ Steinbeis University, Berlin, Germany

\section{References}

1 Benson VS, Patnick J, Davies AK et al. Colorectal cancer screening: a comparison of 35 initiatives in 17 countries. Int J Cancer 2008; 122: $1357-1367$

2 Krebs in Deutschland 2005/2006. Berlin: Häufigkeiten und Trends; 2010

3 Mandel JS, Bond JH, Church TR et al. Reducing mortality from colorectal cancer by screening for fecal occult blood. Minnesota Colon Cancer Control Study. N Engl J Med 1993; 328: 1365-1371

4 Mandel JS, Church TR, Bond JH et al. The effect of fecal occult-blood screening on the incidence of colorectal cancer. N Engl J Med 2000; 343: $1603-1607$

5 Hardcastle JD, Chamberlain JO, Robinson MH et al. Randomised controlled trial of faecal-occult-blood screening for colorectal cancer. Lancet 1996; 348: $1472-1477$
6 Kronborg $O$, Fenger $C$, Olsen $J$ et al. Randomised study of screening for colorectal cancer with faecal-occult-blood test. Lancet 1996; 348 : $1467-1471$

7 Atkin WS, Edwards R, Kralj-Hans I et al. Once-only flexible sigmoidoscopy screening in prevention of colorectal cancer: a multicentre randomised controlled trial. Lancet 2010; 375: 1624-1633

8 Segnan N, Armaroli P, Bonelli $L$ et al. Once-only sigmoidoscopy in colorectal cancer screening: follow-up findings of the Italian Randomized Controlled Trial - SCORE. J Natl Cancer Inst 2011; 103: 1310-1322

9 Schoen RE, Pinsky PF, Weissfeld JL et al. Colorectal-cancer incidence and mortality with screening flexible sigmoidoscopy. N Engl J Med 2012; 366: $2345-2357$

10 Gatta G, Capocaccia R, Sant $M$ et al. Understanding variations in survival for colorectal cancer in Europe: a EUROCARE high resolution study. Gut 2000; 47: $533-538$

11 Becker N. Epidemiological aspects of cancer screening in Germany. J Cancer Res Clin Oncol 2003; 129: 691-702

12 Pox C, Aretz S, Bischoff SC et al. [S3-guideline colorectal cancer version 1.0]. Z Gastroenterol 2013; 51: $753-854$

13 German Guideline of Federal Board of Medical Insurances and Representatives of Private Practice and Hospital Physicians. Richtlinie des Gemeinsamen Bundesausschusses über die Früherkennung von Krebserkrankungen. http://www.g-ba.de [Accessed 17 January 2012]

14 Robert Koch Institut. Beiträge zur Gesundheitsberichterstattung. 20 Jahre nach dem Fall der Mauer: Wie hat sich die Gesundheit in Deutschland entwickelt. Kapitel 5. Prävention und Gesundheitsförderung. S 178. November 2009. http://www.gbe-bund.de [Accessed 17 January 2012]

15 Wuppermann D, Wuppermann U, Riemann JF. [Actual state of knowledge of the German population about the early detection of colorectal cancer - a study by the „Stiftung LebensBlicke“ in cooperation with the Institute for Demoscopy in Allensbach]. Z Gastroenterol 2009; 47: $1132-1136$

16 Creeden J, Junker F, Vogel-Ziebolz S et al. Serum tests for colorectal cancer screening. Mol Diagn Ther 2011; 15: 129-141

17 deVos T, Tetzner R, Model F et al. Circulating methylated SEPT9 DNA in plasma is a biomarker for colorectal cancer. Clin Chem 2009; 55: 1337-1346

18 Grutzmann R, Molnar B, Pilarsky C et al. Sensitive detection of colorectal cancer in peripheral blood by septin 9 DNA methylation assay. PloS ONE 2008; 3: e3759

19 Lofton-Day C, Model F, Devos T et al. DNA methylation biomarkers for blood-based colorectal cancer screening. Clin Chem 2008; 54: $414-$ 423

20 Church TR, Wandell M, Lofton-Day C et al. Prospective evaluation of methylated SEPT9 in plasma for detection of asymptomatic colorectac cancer. Gut 2014; 63: 317-325

21 Ladabaum U, Song $K$. Projected national impact of colorectal cancer screening on clinical and economic outcomes and health services demand. Gastroenterology 2005; 129: 1151 - 1162

22 Sharaf $R N$, Ladabaum U. Comparative effectiveness and cost-effectiveness of screening colonoscopy vs. sigmoidoscopy and alternative strategies. Am J Gastroenterol 2013; 108: 120-132

23 Munich Cancer Registry. http://www.tumorregister-muenchen.de [Accessed 17 January 2012]

24 Statistisches Bundesamt Deutschland. Bevölkerung, Geburten und Sterbefälle, Tabellen. April 2010. http://www.destatis.de/jetspeed/ portal/cms/Sites/destatis/Internet/DE/Navigation/Statistiken/Bevoelkerung/GeburtenSterbefaelle/GeburtenSterbefaelle.psml [Accessed 17 January 2012]

25 Glaeske G, Schicktanz C. BARMER GEK Arzneimittel-Report 2010 http://www.barmer-gek.de/barmer/web/Portale/Presseportal/Subportal/Infothek/Studien-und-Reports/Arzneimittelreport-2010/Arzneimittel-Report-lang,property=Data.pdf [Accessed 17 January 2012]

26 Glaeske G, Höffken K, Ludwig WD et al. Sicherstellung einer effizienten Arzneimittelversorgung in der Onkologie. Gutachten im Auftrag des Bundesministeriums für Gesundheit. Bremen. August 2010. http:// www.bmg.bund.de/fileadmin/redaktion/pdf_allgemein/Gutachten_Sicherstellung_einer_effizienten_Arzneimittelversorgung_in_der_Onkologie.pdf [Accessed 17 January 2012]

27 Goerner M, Riemer-Hommel P. Economic impact of alternative adjuvant chemotherapy regimens for stage III colon cancer. Onkologie 2009; 32: 647-652

28 EBM: Office-based doctor remuneration. KVB: National Association of Statutory Health Insurance Physicians and the regional Associations of 
Statutory Health Insurance Physicians (Kassenärtzliche Bundesvereinigung). March 2011. http://www.kbv.de [Accessed 17 January 2012]

29 InEK - Institute for the Hospital Remuneration System (Institut für das Entgeltsystem im Krankenhaus). German DRGs. March 2011. http:// www.g-drg.de [Accessed 17 January 2012]

30 Hundt S, Haug $U$, Brenner $H$. Comparative evaluation of immunochemical fecal occult blood tests for colorectal adenoma detection. Ann Intern Med 2009; 150: 162-169

31 Whitlock EP, Lin JS, Liles E et al. Screening for colorectal cancer: a targeted, updated systematic review for the U.S. Preventive Services Task Force. Ann Intern Med 2008; 149: 638-658

32 Zauber AG, Lansdorp-Vogelaar I, Wilschut J et al. Technology Assessment: Cost-Effectiveness of DNA Stool Testing to Screen for Colorectal Cancer. Report to AHRQ and CMS from the Cancer Intervention and Surveillance Modeling Network (CISNET) for MISCAN and SimCRC Models 2007. http://www.cms.gov/Medicare/Coverage/DeterminationProcess/downloads/id52TA.pdf [Accessed 3 May 2014]

33 Levin B, Lieberman DA, McFarland B et al. Screening and surveillance for the early detection of colorectal cancer and adenomatous polyps, 2008: a joint guideline from the American Cancer Society, the US Multi-Society Task Force on Colorectal Cancer, and the American College of Radiology. Gastroenterology 2008; 134: 1570-1595
34 Song K, Fendrick AM, Ladabaum U. Fecal DNA testing compared to conventional colorectal cancer ccreening methods: a decision analysis. Gastroenterology 2004; 126: 1270-1279

35 Siegel JE, Weinstein MC, Russell LB et al. Recommendations for reporting cost-effectiveness analyses. Panel on Cost-Effectiveness in Health and Medicine. JAMA 1996; 276: 1339-1341

36 Weinstein MC, Siegel JE, Gold MR et al. Recommendations of the Panel on Cost-effectiveness in Health and Medicine. JAMA 1996; 276: $1253-1258$

37 Lipscomb J, Weinstein MC, Torrance GW. Time preference. In: Gold MR, Siegel JE, Russell LB et al., eds. Cost-effectiveness in health and medicine. New York, NY: Oxford University Press; 1996: 214-235

38 Briggs A, Claxton K, Sculpher M. Making decision models probabilistic In: Decision modelling for health economic evaluation. Cambridge, MA: Oxford University Press; 2006: 77-120

39 Screening for colorectal cancer: U.S. Preventive Services Task Force recommendation statement. Ann Intern Med 2008; 149: 627-637

40 Ladabaum $U$, Allen J, Wandell $M$ et al. Colorectal cancer screening with blood-based biomarkers: cost-effectiveness of methylated septin 9 DNA versus current strategies. Cancer Epidemiol Biomarkers Prev 2013; $22: 1567-1576$

41 van Roon AH, Goede SL, van Ballegooijen M et al. Random comparison of repeated faecal immunochemical testing at different intervals for population-based colorectal cancer screening. Gut 2013; 62: 409-415

\section{Appendix}

online content viewable at: www.thieme-connect.de 